\title{
Editorial
}

\section{Scarless Thyroid Surgery}

\section{Rohaizak M}

Department of Surgery, Faculty of Medicine, Universiti Kebangsaan Malaysia Medical Centre, Jalan Yaacob Latif, Bandar Tun Razak, 56000 Cheras, Kuala Lumpur, Malaysia.

\section{History}

Traditionally, treatment for thyroid diseases is associated with very high mortality and morbidity. Various techniques been performed in the past including finger dissection and the use of finger nails to remove the thyroid tissues. The earliest reported thyroidectomy was probably by Abu al-Qasim alZahrawi or Albucasis. It was mentioned that, he performed an enucleation of a thyroid nodule in the 11 th century in Spain. This was followed by Salerno in the twelfth centuries, using different technique, including setons, hot irons and caustic powders. The first documented partial thyroidectomy was performed by Pierre Joseph Desault in 1791. But at that time, thyroidectomy was considered as a barbaric surgery with high mortality. The poor result and high mortality had lead to the imprisonment of surgeons and total ban of the operation by the French Academy of Medicine in 1850 . The advancement of thyroid surgery was most obvious in the second half of the nineteenth century when Theodor Billroth managed to reduce the mortality dramatically, thanks to the establishment of antisepsis, arterial ligation and precise capsular dissection which was introduced at the same time. There was further improvement on the mortality of the thyroid surgeries to almost zero, but the basic principles remain the same: identification of the recurrent laryngeal nerves and preservation of the parathyroid glands. What becoming a more important issue is the surgery now been performed for smaller thyroid for cosmetic reason, with the need to avoid visible scar in the neck

\section{Minimal Access Thyroid Surgery}

A standard conventional thyroid surgery is performed by making a low collar neck incision, measuring a round $5-6 \mathrm{~cm}$, along the skin crease, $2 \mathrm{~cm}$ above the clavicle. The resultant scar varies from a fine scar to an ugly hypertrophic scar or keloid. Techniques been introduced either to reduce the scar in the neck, or to have hidden scar away from the neck or completely scar less by going through the natural orifice. The first attempt was made by Michele Gagner in 1996 when he performed a minimally invasive parathyroidectomy using gas $\mathrm{CO} 2$ insufflation. The surgery lasted for 5 hours and complicated post operatively by surgical emphysema. Further modification was made by going through the lateral part by JF Henry and central gasless access by Paul Miccoli [Minimally invasive videoassisted thyroidectomy (MIVAT)]. A meta-analysis of MIVAT shows no significant difference in overall morbidity compared to conventional open thyroidectomy. The intra-operative blood loss was also similar between the two groups but the operative time is significantly longer in the MIVAT group (75.2 vs. $59.2 \mathrm{~min}, \mathrm{SMD}=1.246,95 \% \mathrm{CI}=0.227-2.266)$. The advantage of MIVAT is significantly shown in better post-op pain score and patients' satisfactory score about their scar.

\section{Remote Access Thyroid Surgery}

Despite the small scar in the neck, it is still visible and can be disfiguring. Further attempt was made by moving the scar to hidden areas. This provides 
superior cosmesis but more invasive as the surgery will be performed from a remote area. It was first pioneered by Kazuo Shimizu in 1999 by having a $5 \mathrm{~cm}$ infraclavicular incision approaching from anterior as in conventional surgery without gas insufflations. This was followed by more distant access from the breast and axillary approaches. The axillo-bilateral breast approach (ABBA) has limited ability to visualize both lobes, and further modified to bilateral Axillo-Breast approach (BABA) by Choe et al. in 2007 where surgery can be performed as in standard conventional thyroidectomy. Around the same time, Tran Nguc Luong in 2003 introduced the Shoulder Axillary Breast approach which greatly assisted the technique. There was concerned about having the trocar and dissection coming from the breast, Suchart Chatawibul introduced in 2001 a total video-endoscopic thyroidectomy by an axillary approach. The technique was further improvised by performing robotic transaxillary thyroidectomy which was more popular among the Asian compared to Europe. Meta-analysis comparing remote access thyroidectomy to conventional open thyroidectomy, shows it superiority in term of cosmesis but higher rate of transient recurrent laryngeal nerve palsy $[\mathrm{OR}=2.06,95 \% \mathrm{CI}$ (1.28, 3.31), $\mathrm{P}=0.003]$. and longer operative time $[\mathrm{MD}=38.18,95 \% \mathrm{CI}(22.24,54.11), \mathrm{P}<0.00001]$. Other parameters studied such as permanent nerve palsy, permanent hypocalcaemia and disease recurrence are not significantly different.

\section{Natural orifice transluminal endoscopic surgery (NOTES)}

Remote access surgery is successful in moving the scar away from the neck but is a maximally invasive surgery. A large amount of flap dissection is needed in order to reach the thyroid gland. Natural orfices surgery through the maouth is a truly a scarless thyroidectomy. Two approaches were popularized, the sublingual and oral vestibular approaches. The sublingual approach was first performed by Witzel et al in 2007 but the technique was criticized due to the morbidity, caused by dissection of the floor of the mouth such as hypoglossal nerve injury and infection. The oral vestibular approach was first described by Richmon et al. with better outcome and further refined by Anuwong et al. reducing the incidence of mental nerve injury (3). Early results show that the technique can safely be performed in moderate size benign thyroid nodules and small thyroid cancer. Surprisingly, the morbidity from infection is very low and the use of an intra-operative nerve monitoring has a promising result in reducing further the already low incidence of recurrent laryngeal nerve injury.

\section{Summary}

Thyroidectomy for benign and malignant diseases has progressed dramatically over the last two decades. Moving from large collar incision to no scar is a very good news to the patient with neck swellings. The morbidity of the surgery remains low regardless of the technique and approaches used but scarless surgery is still limited to benign diseases and small cancers. Further study and future refinement of the technique might make these techniques also applicable to large tumours.

\section{References}

1. Zhang P, Zhang HW, Han XD, Di JZ, Zheng Q. Meta-analysis of comparison between minimally invasive video-assisted thyroidectomy and conventional thyroidectomy. Eur Rev Med Pharmacol Sci 2015; 19(8): 1381-7.

2. Li Y, Zhou X .Comparison between endoscopic thyroidectomy and conventional open thyroidectomy for papillary thyroid microcarcinoma: A meta-analysis. J Cancer Res Ther 2016; 12(2): 550-5

3. Anuwong A, Kim HY, Dionigi G. Transoral endoscopic thyroidectomy using vestibular approach: updates and evidences. Gland Surg 2017; 6(3): 277-84. 